\title{
Phase behavior and interfacial curvature in water-oil-surfactant systems Reinhard Strey
}

The phase behavior of water-oil-surfactant systems follows general patterns. The driving force for the phase behavior has been identified as the variation of the mean curvature of the surfactant film with temperature. The minimum of interfacial tension accompanying the phase inversion has been shown to be caused by the mean curvature passing zero. These findings help increase our understanding of investigations in a number of other areas including the role of cosurfactants, phase inversion temperature, and hydrophilic-lipophilic balance for emulsion formation. Furthermore, new applications of microemulsions as reaction media and new formulations all make use of tuning the curvature of the amphiphilic film.

\section{Address}

Max.Planck-Institut für Biophysikalische Chemie, Postfach 2841, D-37018 Göttingen, Germany; e-mail: rstrey@gwdg.de

Current Opinion in Colloid \& Interface Science 1996, 1:402-410

(C) Current Science Ltd ISSN 1359-0294

\begin{tabular}{|c|c|}
\hline \multicolumn{2}{|c|}{ Abbreviations } \\
\hline $\mathrm{ACN}$ & alkane carbon number \\
\hline AOT & Sodium-di(2-ethylhexyl)sulfosuccinate \\
\hline $\begin{array}{l}C_{j} E_{j} \\
D D A B\end{array}$ & $\begin{array}{l}\text { n-alkyl polyglycolether with i methylene and j oxyethylene groups } \\
\text { didodecy!dimethylammonium bromide }\end{array}$ \\
\hline DTAB & dodecyltrimethylammonium bromide \\
\hline FA & formamide \\
\hline HLB & hydrophilic-lipophilic balance \\
\hline OP & optimal point \\
\hline PIT & phase inversion temperature \\
\hline SANS & small angle neutron scattering \\
\hline
\end{tabular}

\section{Introduction}

Mixtures of water, oil and surfactant (s), and their phase behaviors have been under study for several decades. The addition of surfactant molecules to water and oil may lead to the formation of emulsions, microemulsions and lyotropic mesophases. In all these cases, the water- and oil-rich domains are separated by an amphiphilic film. The nature of the amphiphilic film at the microscopic level has been the subject of intensive research, as its propertics are decisive for the formation of emulsions, microemulsions and lyotropic mesophases. A very useful theoretical treatment of water-oil-surfactant systems has been given by Gompper and Schick [100]. A literature survey of paper titles and abstracts reveals that since the beginning of 1995,600 papers have appeared on the subject of microemulsions, 1500 on emulsions, 500 on mesophases, and 400 with water-oil-surfactant systems, of which 30 relate to the phase behavior in particular. Another 400 papers deal with surfactant or amphiphilic interfaces, and 40 with the water-oil interfacial tension. These papers constitute an enormous flood of information, which the reader is likely to survive only if he has some strategy as to how to proceed. All I can do is offer my personal current opinion.

\section{Microemulsions}

Microemulsions are thermodynamically stable, macroscopically homogeneous mixtures of water, oil and surfactant. Microscopically, a surfactant film separates the two incompatible solvents. In this review, we recall the experimentally observed interfacial curvature of the film, as it expresses itself in a systematic metamorphosis of the microstructures and phase behavior, when a suitable tuning parameter is varied [2*0]. As such, in the past, temperature, pressure, fourth and fifth components have been applied. Ever since the pioneering work of Winsor [3], it has been known that the general phase behavior proceeds as shown schematically by the test tubes on the left-hand side of Figure 1. We discuss here, as model systems, non-ionic surfactants of the n-alkyl polyglycolether rype. The right hand side of Figure 1 relates the interfacial tensions to the phase diagrams.

\section{Origin of the phase behavior}

Winsor.[3] discussed, qualitatively, how a mixture containing a sufficient amount of surfactant and equal amounts of water and oil may separate into a water-rich microemulsion in equilibrium with an excess oil phase (Fig. 1, 2, Winsor I), an oil-rich microemulsion in equilibrium with an excess water phase (Fig. 1, $\overline{2}$, Winsor II), or into three phases with a middle phase microemulsion in simultaneous equilibrium with excess oil and water phases (Winsor III). The progression is understood as a result of the change in the curvature of the amphiphilic film driven by a variation of the interactions on either side of the film. Winsor worked at constant temperature using five-component systems including salt and an ionic surfactant-cosurfactant mixture. Accordingly, he tuned the curvature of the films by the salt concentration and/or the surfactant/cosurfactant ratio (see below for the role of the cosurfactant in the film).

Even during 1995, 60 papers appeared using cosurfactant in their formulations. If one does not have the choice of optimal surfactants (there may be various commercial or scientific reasons), it may be helpful to use these additives (c.g. in emulsion formulations, see below). Quantitative measurements of structures and their interpretation is facilitated, however, if one restricts oneself to the three components really needed - a polar, a non-polar and an amphiphilic substance - and uses temperature to tune the phase behavior. Knowledge obtained by this procedure can then be used with success to evaluate other systems. 


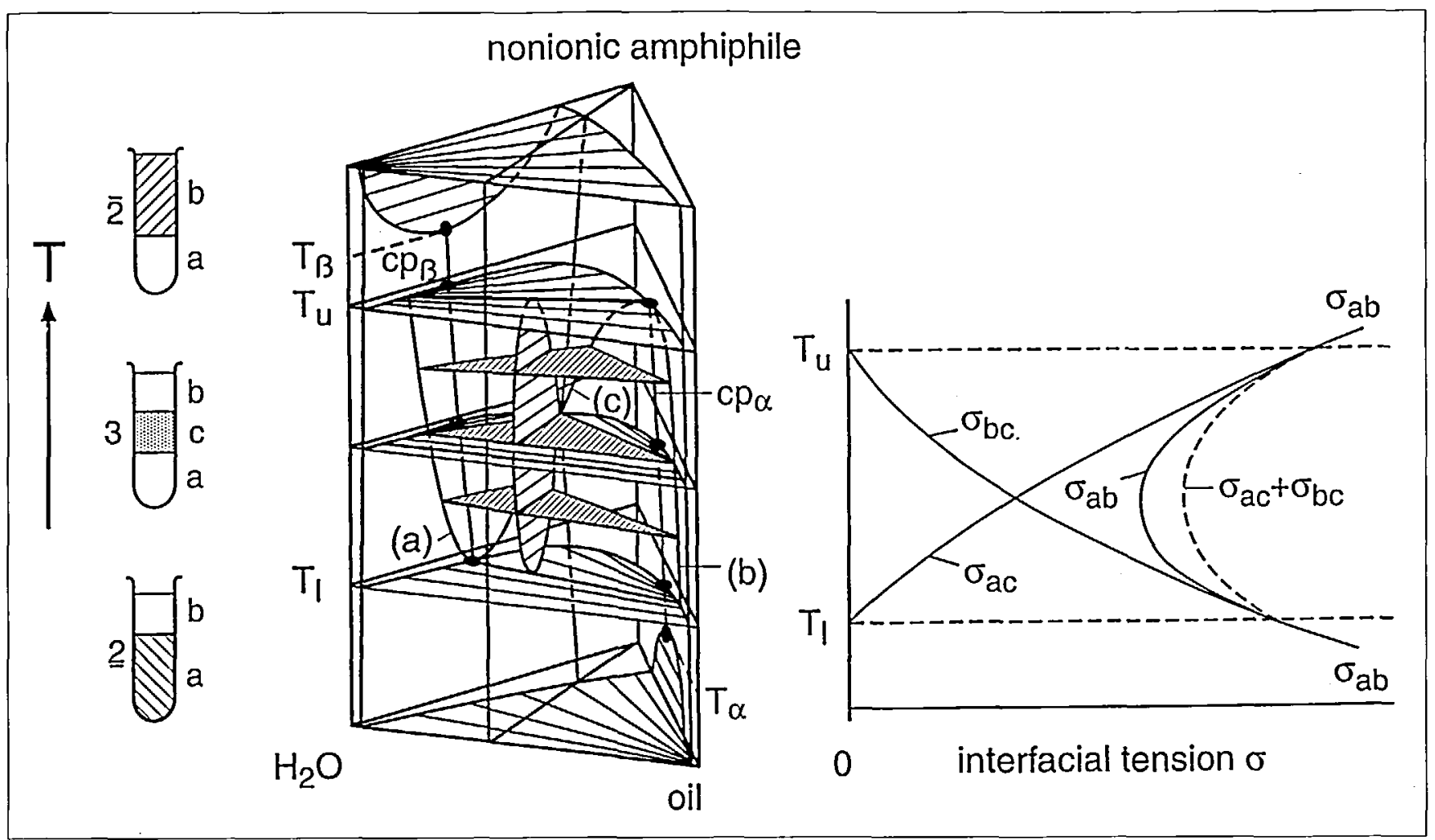

The phase behavior of water-oil-surfactant systems (schematic) with temperature. The symbols (a), (b) and (c) denote the water-rich, oil-rich and amphiphile-rich phase, respectively. $T_{1}$ and $T_{u}$ are the lower and the upper temperatures of the three phase body. Critical lines ( $c_{p}$ ) originate from the lower critical point of the binary water-amphiphile system at $T_{\beta}$, and from the upper critical point of the oil-amphiphile system at $T_{\alpha}$. The triangles illustrate the coexistence of the three phases (a), (b) and (c) at various temperatures. The interfacial tension minimum of $\sigma_{a b}$ is seen to arise from the monotonic behavior of $\sigma_{a c}$ and $\sigma_{b c}$ and the stability condition $\sigma_{a b} \leq \sigma_{a c}+\sigma_{b c}$. Taken with permission from [44].

Quite generally, a basic study starts with the phase behavior first. Once one has established the correct phase behaviour, the phase volume, optical appearance, position in the test tube, and so on provide the first hints as to the composition and structure of the phases. Within the past decade, Kahlweit and colleagues [4-7] have systematically studied the phase behavior of the n-alkyl polyglycolether system as it depends on temperature, the nature of the oil and the hydrophilic and hydrophobic groups of the surfactant molecules. The basic features of this system were described as early as 1985 in a review [4], which even today may be recommended as a starting point. The subsequent investigation of the chain-length dependence [5] demonstrated that long-and short-chain surfactants behave similarly and that some properties are common to the three-phase bodies [6]. These workers also showed how the behavior of ionic surfactants differs from that of non-ionic surfactants in terms of the reverse temperature dependence [7], enabling the formation of a temperature-insensitive microemulsion, a feature still of interest today $\left[8^{\circ}, 9\right]$. Finally, in a qualitative thermodynamic description the essential features $[10,11]$ of the phase behavior of microemulsion systems were summarized [12].
A quantitative confirmation of Winsor's views has only recently been performed [2*0] by tuning the curvature of the amphiphilic film with temperature. Given the importance of the observed trends for the field as a whole, we shall recall hereafter the most important features. We refer the reader, however, to the original paper for more details.

\section{Mean curvature}

It is crucial to realize that the area-averaged mean curvature $\langle H\rangle$ is temperature-dependent. It is defined as:

$$
<H>=\frac{c_{1}+c_{2}}{2}
$$

where $c_{1}$ and $c_{2}$ are the principal curvatures. As the temperature is tuned away from the balanced state, where the mean curvature $\langle H\rangle=0$, the microemulsions take up either more oil, as the temperature is increased, or more water as temperature is decreased. The temperature dependence of $\langle H\rangle$ is shown in Figure 2a for the water-n-octane- $\mathrm{C}_{12} \mathrm{E}_{5}\left(\mathrm{C}_{\mathrm{i}} \mathrm{E}_{\mathrm{j}}\right.$, n-alkyl polyglycolether with $\mathrm{i}$ methylene and $\mathrm{j}$ oxyethylene groups) system [2०0]. Note that $H$ and $c_{i}$ (shown as dashed lines) are counted as 
positive if oil is enclosed and negative if water forms the interior, the latter case corresponding to inverse micelles. Examples involving the use of inverse micelles are described below. For droplet structures, $\langle H\rangle \approx\left\langle c_{1}\right\rangle \approx\left\langle c_{2}\right\rangle$ $\approx 1 /\langle r\rangle$, where $\langle r\rangle$ is the mean radius of the droplets. For bicontinuous microemulsions, where $\langle H\rangle$ becomes zero, the individual $c_{i}$ values remain finite. At the mean temperature of the three-phase region $T_{m} .\left[\approx\left(T_{u}+T_{l}\right) / 2\right],<$ $\left.c_{1}\right\rangle \approx-\left\langle c_{2}\right\rangle$, so that the principle curvatures necessarily become zero for different temperatures. It turns out that one has to postulate that $\left\langle c_{1}\right\rangle$ and $\left\langle c_{2}\right\rangle$ become zero near $T_{u}$ and $T_{b}$, respectively, where locally cylindrical structures have been observed. Of course, the linear dependence is only a first order approximation (in reality the curvatures are more likely to vary sigmoidally $\left.\left[2^{\circ} \cdot\right]\right)$, but it suffices to discuss the linear approximation $\langle H\rangle=c\left(T-T_{m}\right)$ where $c=10^{5} \mathrm{~cm}^{-1} \mathrm{~K}^{-1}$.

\section{Characteristic length scale}

As $\langle H\rangle$ passes zero, the characteristic length scale, that is, the radius of the droplets exhibits a maximum. In Figure $2 \mathrm{~b}$, the maximum is shown, where the full line is calculated from $[2 \cdot \bullet]$ :

$$
\xi=1 / \sqrt{\frac{1}{2}\left(c_{1}^{2}+c_{2}^{2}\right)} .
$$

It is important to realize that the maximum in the length scale appears as a consequence of the mean curvature passing through zero while the principle curvatures remain non-zero.

\section{Origin of the ultra-low interfacial tension}

As a further consequence of the length scale reaching a maximum, the interfacial tension between the water and oil phases passes through a minimum. This can simply be explained by considering that dispersion of one medium in another will occur if the free energy cost of forming the surface of an object is:

$$
\sigma_{a b} \xi^{2} \approx k T
$$

where $\sigma_{a b}$ is the interfacial tension. Figure $2 c$ depicts the variation in $\sigma_{\mathrm{ab}}$ with temperature. A discussion in terms of the bending energy (first proposed by Helfrich [13]) being the only relevant contribution yields $[2 \bullet, 14 \bullet]$

$$
\sigma_{a b}=2 H^{2}(\kappa+\bar{\kappa})+\bar{\kappa} c_{1} c_{2}
$$

where $\kappa$ and $\bar{\kappa}$ are the splay and saddle-splay elastic constants, respectively. The full line in Figure $2 \mathrm{c}$ is calculated from Equation 4. It is evident that this simple set of equations describes the trends rather well. Evidently, the spontaneous curvature $H_{0}$ and the splay and saddle-splay elastic constants $\kappa$ and $\bar{\kappa}$ are suffice to capture the gross features.

Accordingly, the phase behavior may be understood in terms of a temperature-dependent spontaneous curvature

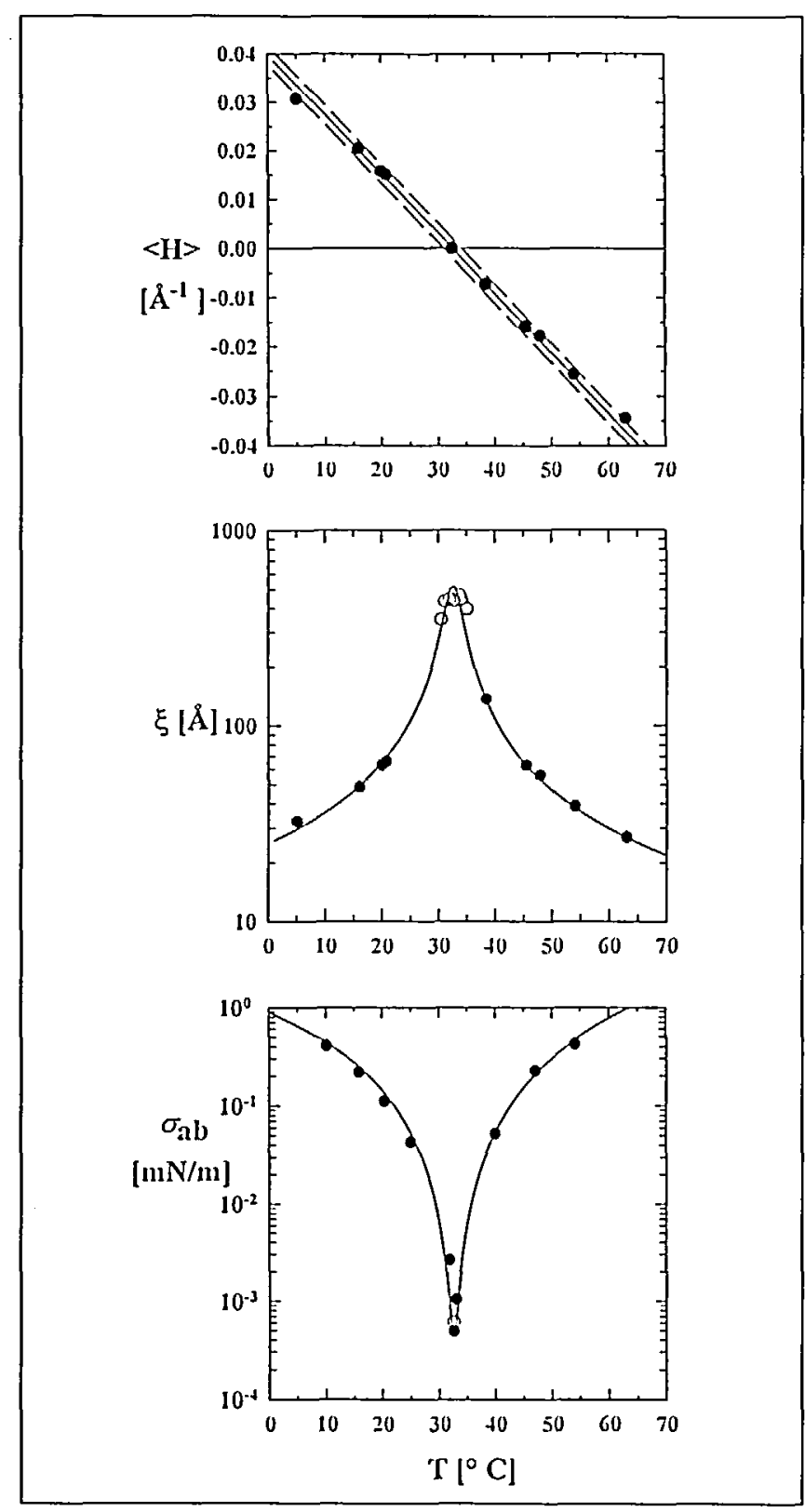

Illustration of the most important parameter variations for microemulsions. Because the mean curvature $\langle H\rangle$ [and also the principal curvatures $c_{1}, c_{2}$ (dashed lines)] varies nearly linearly and passes through zero, the length scale $\xi$ reaches a maximum (note the inverse relatirn of $H$ and $\xi$ ). As a further consequence $\sigma_{a b}$ reaches a minimum, because $\sigma_{a b} \xi^{2}=k T$. Taken with permission from [2*•].

driving the phase behavior, which then forces the interfacial tension through the extreme minimum.

\section{Role of alcohols in microemulsions}

If the surfactants were not optimized, alcohols were sometimes added. Early on, Winsor [3] considered alcohols ('cosurfactants') as indispensable ingredients for the formation of microemulsions. Shinoda had already, however, shown that microemulsions can be made without alcohols 
using only three components: water, a hydrocarbon and a single non-ionic surfactant (e.g. polyethylene glycol ether). This proves that alcohols are not a prerequisite for the formation of microemulsions.

Recently, a study on microemulsions made using longchain amphiphile mixtures of $\mathrm{C}_{12} \mathrm{E}_{2}$ and $\mathrm{C}_{12} \mathrm{E}_{8}$ and mixtures of hexanol (denoted formally as $\mathrm{C}_{6} \mathrm{E}_{0}$ ) and $\mathrm{C}_{12} \mathrm{E}_{8}$ at constant temperature $\left(25\right.$ or $\left.35^{\circ} \mathrm{C}\right)$ quantified the partitioning of alcohols between oil and amphiphilic films [15*0]. The effect of molecular mass of the oil on the three-phase behavior and maximal solubilization was investigated. It was found that the minimum weight fraction of surfactant required to yield equal weights of water and oil in a single phase increases with increasing molecular mass of the hydrocarbon oil. Non-ionic surfactants mainly distribute between oil domains and the interface inside the microemulsion phase. Only those surfactant molecules that reside at the interface accomplish solubilization. As Kunieda et al. [15'?] have demonstrated, the mixing ratio of the surfactants at the interface is obtainable from the geometric relation of the three-phase tie triangle in the exact composition tetrahedron. The net solubilizing power is found to dramatically decrease with increasing molecular mass of the oil.

In a related study, Penders and I [16*0] studied the influence of n-octanol (denoted as $\mathrm{C}_{8} \mathrm{E}_{0}$ ) on the phase behavior of the ternary microemulsion system water-noctane $-\mathrm{C}_{8} \mathrm{E}_{5}$. From order parameter profile measurements in a previous study [17] it was expected that the alcohol molecules would arrange themselves in the film with the $\mathrm{OH}$ group oriented towards the aqueous subphase. The alcohol achieves predominantly two effects. The first is to change the effective hydrophilicity of the amphiphilic mixture $\mathrm{C}_{8} \mathrm{E}_{5}+\mathrm{C}_{8} \mathrm{E}_{0}$, that is, the spontaneous curvature of the film is adjusted by the mixing ratio just as by the temperature (Fig. 2). The second effect is that the addition of $\mathrm{C}_{8} \mathrm{E}_{0}$ to the ternary system $\mathrm{H}_{2} \mathrm{O}$-n-octane- $\mathrm{C}_{8} \mathrm{E}_{5}$ increases the strength (or solubilization capacity) of the amphiphilic mixture. An increased efficiency or amphiphilicity can quite generally be related to a decrease in interfacial tension between bulk water- and oil-rich phases $[1 \bullet, 2 \bullet \bullet, 5]$.

\section{Synergism}

Penders and I [ $\left.16^{\circ}\right]$ have compared the interfacial tensions in the quaternary water-n-octane- $\mathrm{C}_{8} \mathrm{E}_{5}-\mathrm{C}_{8} \mathrm{E}_{0}$ system to interfacial tension measurements of the type shown in Figure 2c. It was shown that a synergism of the $\mathrm{C}_{8} \mathrm{E}_{5}-\mathrm{C}_{8} \mathrm{E}_{0}$ couple yields a tension four fold lower than that of the equivalent single component surfactant. The phase behavior of the quaternary microemulsion system $\mathrm{H}_{2} \mathrm{O}$-n-octane- $\mathrm{C}_{8} \mathrm{E}_{5}-\mathrm{C}_{8} \mathrm{E}_{0}$ described in [16*0] has its roots in the phase behavior of the ternary (or rather pseudo-binary) system $\mathrm{H}_{2} \mathrm{O}-\mathrm{C}_{8} \mathrm{E}_{5}-\mathrm{C}_{8} \mathrm{E}_{0}$ described in an earlier study $\left[1^{\circ}\right]$. For the latter system (with no oil present), we found that the combination of the two short-chain molecules $\mathrm{C}_{8} \mathrm{E}_{5}$ and $\mathrm{C}_{8} \mathrm{E}_{0}$ initiates the formation of mesophases, such as the (dilute) lamellar phase $\mathrm{L}_{\alpha}$, and the sponge-like bilayer phase $\mathrm{L}_{3}$, which normally occur only for longer-chain surfactants such as $\mathrm{C}_{12} \mathrm{E}_{5}$. Accordingly, a synergistic effect was also seen in the binary system $\left[18^{\circ}\right]$.

In general, one can say that alcohols are a useful, easyto-handle additive. It is not an indispensable additive but may make life easier where unknown surfactants need to be examined (c.g. [19]).

\section{Mesophases}

Mesophases usually occur at higher surfactant concentrations. Interestingly they follow the same systematic trends of the curvature of the film. Leaver et al. [20 ] have examined a section of the composition-temperature phase prism of a ternary non-ionic surfactant-water-oil system defined by a constant surfactant/oil ratio. The structural sequence of normal spheres to planar bilayers, via cylinders, is observed as a function of decreasing water concentration at fixed temperature. As a function of increasing temperature at fixed surfactant and oil concentration, the authors note the change in sign in mean curvature of the polar/apolar interface similarly as discussed above. The mean curvature progressively alters from positive values in the low temperature microemulsion phase to negative mean curvature in the high temperature $\mathrm{L}_{3}$ phase, with an intermediate lamellar phase where the mean curvature passes through zero. The phase diagram of this section is similar to that of the binary surfactant-water system. A micellar cubic phase is observed between the microemulsion and the hexagonal phase but no bicontinuous cubic phase is observed.

A related study on the phase behavior of cubic liquid crystals has examined the mesophases of a system of single chain ionic surfactant, short chain alcohol, and comparable amounts of oil and brine. Studying the small angle $\mathrm{X}$-ray diffraction, deGeyer [21] focused on two cubic liquid crystal phases of probable space groups $\mathrm{Pm} 3 \mathrm{n}$ and $\mathrm{Fd} 3 \mathrm{~m}$. The structures of both cubic mesophases correspond to an aqueous film forming a network of polyhedral cells (with oil interior and hydrophilic walls).

The case of the swelling of sponge-like bicontinuous mesophases of bilayers of surfactant (or lipid) in water [22*] has been analytically analyzed with formulas for the swelling derived both for the constant aggregate thickness case and fixed surface area per surfactant molecule at an imaginary surface located within the bilayer. Approximate swelling laws were compared with swelling behavior of disconnected sheet-like, rod-like and globular aggregates. Sponge-like ('oil-in-water'[O/W]) bicontinuous aggregates can be readily distinguished from rods, sheets or globules in surfactant-lipid aggregates; the morphologies of aggregates of reversed curvature ('water-in-oil'[W/O]) are less easily deduced from swelling data. 


\section{Emulsions}

Emulsions applied in cosmetics and for technical applications must fulfill certain requirements concerning fine dispersity and long-term stability. In practice, besides mechanical energy input, the phase behavior of the emulsion systems may be utilized to obtain optimal emulsification results. (O/V) emulsions stabilized with non-ionic emulsifiers may show temperature-induced phase inversion from an (O/W) to a (W/O) type, just as do the microemulsions discussed above. Often; during phase inversion a microemulsion phase is passed, which is connected to the ultra-low interfacial tensions. Because of this ultra-low interfacial tension, finely dispersed emulsions with long-term stability can be produced by phase-inversion emulsification without high energy input. The important basic properties of the fatty alcohol ethoxylates in water-oil systems and their effects on emulsifying behavior have been reviewed by Foerster et al. [23०]. Systematic study of emulsion and microemulsion phase behavior has established certain rules, which allow the preselection of optimal emulsification routes and optimal emulsifier-oil combinations. On the basis of these rules, an algorithm that enables the calculation of optimal emulsified mixcures for cosmetic and technical emulsions was described.

\section{Emulsion stability}

The correspondence between the equilibrium phase behavior of water-oil-surfactant mixtures and macroemulsion type stability has been studied by Kabalnov et al. $\left[24^{\circ}\right]$. With the insights gained from Figures 1 and 2 their results become easier to understand. These authors argue that both phase behavior and emulsion stability depend on the bending elasticity of the surfactant monolayer at the oil/water interface. At positive spontaneous curvatures $H_{0}$, $(\mathrm{O} / \mathrm{V})$ emulsions are stable, and at negative spontaneous curvatures, (W/O) emulsions are stable; in the balanced state of the surfactant film, where $H_{0}=0$, an emulsion break usually occurs for a wide variety of systems. To explain the effect of the monolayer bending properties on the macroemulsion stability, thermally activated rupture of emulsion films was studied theoretically [24*0]. The authors considered emulsion films covered by saturated surfactant monolayers with strong lateral interactions among the adsorbed surfactant molecules. The monolayer at the edge of a nucleation hole in the emulsion film is strongly curved and the bending energy penalty (c.f. Eq 4) involved leads to a strong dependence of the coalescence barrier on the sign and the absolute value of the monolayer spontaneous curvature $H_{0}$. At large positive spontaneous curvatures, the $\mathrm{O} / \mathrm{W} / \mathrm{O}$ films are stable, with a coalescence barrier (in the first approximation) proportional to the splay modulus. The W/O/W films break without a barrier. Conversely, for large negative values of $H_{0}$, W/O/N films are stable and $\mathrm{O} / \mathrm{N} / \mathrm{O}$ films break without a barrier. In the vicinity of the balanced state, a very steep change in film stability with $H_{O}$ is predicted. The model reproduces the macroemulsion stability observed experimentally, as it depends on the splay moduli.

\section{Hydrophilic-lipophilic balance}

In an interesting review article, Davis [25*0] has mentioned that Bancroft's rule of thumb, that a surfactant which preferentially partitions into water favors the formation of $(\mathrm{O} / \mathrm{V})$ emulsions, and that a surfactant which preferentially partitions into oil favors the formation of $(\mathrm{W} / \mathrm{O})$ emulsions often works well. Because certain combinations of the surfactant hydrocarbon and ethylene oxide chain lengths favored $\mathrm{O} / \mathrm{V}$ or $\mathrm{IV} / \mathrm{O}$ emulsions, the emulsion chemist will choose a water-soluble surfactant to make an $\mathrm{O} / \mathrm{V}$ emulsion and an oil-soluble one to make a W/O emulsion.

To try to put this principle of hydrophile-lipophile balance (HLB) on a quantitative footing, Griffin long ago introduced the concept of an HLB number. According to Griffin, the HLB is the balance of the size and strength of the hydrophilic and lipophilic moieties of a surfactant molecule. The HLB number is based on the molecular groups composing the surfactant. According to this concept, the optimal surfactant for a desired emulsion of a given oil can be chosen by a simple calculation based on data found in the literature.

One problem with the HLB concept is that the HLB numbers are based on ambient temperature data. As shown in Figure 2, a given ethoxylated surfactant can form $\mathrm{O} / \mathrm{W}$ emulsions at room temperature but $\mathrm{W} / \mathrm{O}$ emulsions at higher temperatures. Thus the HLB cannot be related solely to the molecular groups of the surfactant. Temperature interactions with the aqueous and oil phases must be incorporated into the HLB number if it is to be a predictive tool. In addition, a strong correlation has been observed between the formation of an $\mathrm{O} / \mathrm{W}$ emulsion (or a $\mathrm{W} / \mathrm{O}$ ) and whether the surfactant tends to form an aqueous micellar phase or an oil-rich inverted micellar phase. Thus, relating the HLB number to the free energies of micellization in water and oil phases ought to result in a more predictive tool, and it is preferable to introduce an HLB temperature-based on the phaseinversion temperature (PIT), where $H_{0}=\langle H\rangle=0$. This temperature is determined from the water-oil-surfactant phase diagram (e.g. Fig. 1). For non-ionic surfactants, the PIT is the temperature below which a surfactant partitions preferentially into the water phase as oil-swollen micelles and above which it partitions preferentially into the oil phase as water-swollen inverted micelles. The advantage of basing the HLB on the PIT is that the surfactant-oil-water interactions are automatically incorporated into the HLB criterion.

\section{HLB, PIT and $T_{m}$}

The effect of a cosurfactant, frequently needed for stable emulsions similarly can be accounted for as a shift in the PIT. Basing the HLB temperature on the PIT 
shows the strong correlation between emulsion type and ternary or pseudo-ternary phase behavior. Davis [25*0] has explored the connection between the generic ternary phase behavior and emulsification tendencies. The trend from preferential partitioning of surfactant into the aqueous phase, through a balanced or optimal microemulsion (equal uptake of oil and water) to preferential partitioning into the oil phase can be accomplished by a large number of variables [c.g. the field variables temperature, pressure, salinity and alcohol activity, and extended field variables such as the average carbon number of the oil (ACN), the average carbon number of the hydrophobic moiety of the surfactant, the average number of ethylene oxide groups of the hydrophilic moiety of the surfactant, etc.] for the various types of surfactant. The field variable at which the optimal microemulsion occurs provides an optimal point (OP) that can serve as the HLB for a given system. If the field variable is set on one side (above or below depending on the variable) of the OP for a system, the surfactant serves as a hydrophilic agent (favors $\mathrm{O} / \mathrm{W}$ emulsions), and if it is set on the other side of the OP, the surfactant serves as a hydrophobic agent (favors W/O emulsions). The relationship between generic phase behavior of water, oil and surfactant systems has been discussed $\left[1 \bullet \cdot 2 \bullet \bullet, 3-7,8^{\bullet}, 9-12\right]$ and the curvature properties $[2 \cdot \bullet$ ] of surfactant interfacial films. It comes as no surprise that Davis' OP is exactly that point on the temperature scale $T_{m}$ where $H$ becomes zero, $\xi$ reaches a maximum, and $\sigma_{\mathrm{ab}}$ reaches a minimum (Fig. 2).

\section{Microemulsions as reaction media}

Microemulsions provide an interesting application as reaction media because of their local compartmentalization into hydrophilic, hydrophobic and amphiphilic subphases. An example of how the phase behavior may be studied is provided in a paper by Lusvardi et al. [26'], who have examined the microstructure of polymerizable alkyl methacrylates $\left(\mathrm{C}_{\mathrm{k}} M A, \mathrm{k}=1,4\right.$, and 6) in aqueous solutions by applying the cationic surfactants dodecyltrimethylammonium bromide (DTAB) and didodecyldimethylammonium bromide (DDAB). Mixing of the surfactants generates a region in the water-rich corner of the phase diagram where a homogeneous microemulsion phase forms for three alkyl methacrylates of differing hydrophobicity. Small angle neutron scattering (SANS) showed that the size of the O/V microemulsions depends on the DTAB/DDAB ratio and the oil content. Here, the DTAB/DDAB ratio plays the role of the curvature determinant.

Schwuger $e t$ al. [27॰] have elucidated the basic properties of microemulsions and their relation to some already established applications, including phase behavior of microemulsions and interfacial tension between water and oil phases, and applications such as enhanced oil recovery, liquid-liquid extraction, extraction of chemicals from contaminated soils, lubricants and cutting oils, pharmaceuticals and cosmetics, washing, and impregnation and textile finishing. These authors also discussed chemical reactions in microemulsions, including nanoparticle preparation, biochemical reactions, electrochemical and catalytic reactions, polymerization, organic reactions, an example of a reaction process in a microemulsion, and the comparison with phase transfer catalysis.

An example where actual reactions have been carried out in microemulsions is provided by Eastoe and Cox [28]. Droplet-like structures of polydisperse spheres (quantum-sized lead(II) sulfide particles having a mean radius of $1.7 \mathrm{~nm}$ ) have been synthesized using two different reversed micellar media, containing either sodium di(2-ethylhexyl)sulfosuccinate [ $\left.\mathrm{Na}\left(\mathrm{AO}^{\prime} \mathrm{T}\right)\right]$ only or mixed $\mathrm{Na}(\mathrm{AOT}) / \mathrm{Pb}(\mathrm{AOT})_{2}$ systems.

Enzyme reactions can be carried out efficiently in $\mathrm{W} / \mathrm{O}$ microemulsions and reversed micelles. Oldfield [29.] has discussed and reviewed the topics of phase behavior, structure, and dynamics of microemulsions, and the properries and behavior of microemulsified enzymes, as well as recent advances in process engineering of microemulsions with industrial biotransformations, new research trends, and furure prospects. Other studies have examined the morphological characteristics of the enzymatic synthesis of phenolic polymers in the microstructural environment of W/O microemulsions or reversed micelles [30] and possible applications of microemulsion gels [31].

Singh and Shah [32] have explored the enzymatic synthesis of glycerol and fatty acid in monolayers and microemulsions. In monolayers, the esterification reaction yielded triglyceride and monoglyceride as the major products, even though the lipozyme was 1,3-specific. The same reaction was carried out in W/O microemulsions with AOT as surfactant, and monoglyceride and diglyceride were obtained as the major products. The results can be explained on the basis of residence time of the product at the interface, curvature of the interface, orientation of substrate at the interface, and the interfacial activation effect on the lid of the active site of the enzyme.

\section{New surfactants: mixtures and formulations}

Some remarkable new formulations have been reported, replacing either the water, the oil or the surfactant (mixtures). With regard to the latter, the normal Winsor phase behavior has been discussed above. In some cases, however, the IVinsor II phase is not reached by increasing the alcohol content, and a retrograde transition to Winsor I is exhibited instead. Such an anomalous case has been analyzed by Salager $e t$ al. [33०] for a system containing a commercial non-ionic polyethoxylated surfactant, $\mathrm{n}$-heptane, $\mathrm{H}_{2} \mathrm{O}$, and $\mathrm{n}$-pentanol. High pressure liquid chromatography analysis yielded the surfactant oligomer partitioning between phases affected by the alcohol content. The retrograde transition was shown to come from the strong increase in the partitioning of lipophilic and balanced oligomers into the oil phase, with the remaining 
surfactant, in particular the interfacial mixture, becoming more hydrophilic. An important mechanism that should be accounted for if technical grade surfactants and mixtures are to be considered.

A surfactant mixture of dodecyl polyglucosides (with an average of 1.8 glucose units) and a branched alkyl glycerol monoether which acts as a cosurfactant form a balanced microemulsion when only $4 \mathrm{wt} \%$ of this mixture is added to water and cyclohexane [34]. Any water/hydrocarbon ratio can be achieved by varying the surfactant/cosurfactant molar ratio, and tuning the microstructure this way. Investigations of the microemulsion microstructure based on the nuclear magnetic resonance self-diffusion technique have provided evidence for the usual changes in the ratio of W/O self-diffusion. As for the non-ionics discussed above, the microemulsion is of the $\mathrm{O} / \mathrm{N}$ type at high surfactant/cosurfactant molar ratios and of the WV/O type at lower ratios. At intermediate surfactant/cosurfactant ratios, the HLB of the surfactant mixture is balanced and a bicontinuous microemulsion with a low mean curvature surfactant layer is formed. At intermediate oil/water ratios, the microemulsion microstructure changes dramatically with small changes of the surfactant/cosurfactant ratio, just as the non-ionics do with temperature.

Some extremely large oils, such as long-chain alkanes or triglycerides, are difficult to microemulsify. The solubilization of such oils and water in a microemulsion can be improved by the introduction of a so-called extended-surfactant lipophilic linker [35] which has a polypropylene oxide chain inserted between the conventional alkyl ether and ether sulfate groups. This compound, a polypropylene oxide monododecyl ether sulfate, has the general structure $\mathrm{C}_{12} \mathrm{H}_{25}\left(\mathrm{OC}_{3} \mathrm{H}_{6}\right)_{6-14}-\left(\mathrm{OCH}_{2} \mathrm{CH}_{2}\right)_{2} \mathrm{OSO}_{3}-\mathrm{Na}$. These surfactants exhibit a critical micelle concentration and a cloud point that changes with the number of propylene oxide groups per molecule, and show three-phase behavior at optimum formulations with hexadecane, ethyloleate and triglycerides (e.g. soya oil). Values of the optimum solubilization were $-20 \mathrm{wt} \%$ of surfactant for a $1: 1$ water/oil mixture. The results might find application in enhanced petroleum recovery.

At the other extreme, very light oils, such as ethane, propane and butane, require elevated pressures to be liquid [36]. Nevertheless, the phase behavior of non-ionic ethoxylated surfactant-light alkane- $\mathrm{H}_{2} \mathrm{O}$ systems has been examined [37] in detail. In compressible liquids (e.g. propane), phase transitions that normally are induced by changing temperature or salinity can also be accomplished with pressure. A complete transition from a lower $\rightarrow$ middle to upper phase microemulsion with pressure has been reported [37] for the first rime in propane. The phase behavior as a function of the $\mathrm{ACN}$ of the oil component reverses in the light alkanes butane, propane, and ethane. This pattern occurs in both reverse-micelle systems (small water/oil ratio) and Winsor III microemulsions. As for the usual ternary phase diagrams [4] (Fig. 1), the observed pressure and ACN effects can be explained in terms of the miscibility gaps of the binary phase diagrams, and are related to enthalpic and entropic interactions between the surfactant tails and the compressible solvent.

Microemulsions in which water is replaced by another polar protic solvent [e.g. formamide (FA)] was studied [38]. Because hydrocarbons are slightly more soluble in FA than in water, the repulsive hydrophobic interaction between the hydrocarbon tails of the surfactants and FA is found to be weaker than in water. As a consequence, both the mutual solubility and the critical micelle concentration increase considerably upon replacing water by FA. This can be compensated by increasing the carbon number of the tails of the surfactants. With the non-ionic $\mathrm{C}_{\mathrm{i}} \mathrm{E}_{\mathrm{j}}$, one has to increase the carbon number $i$ by 5 , whereas with ionic amphiphiles one must proceed from single-tailed to double-tailed amphiphiles (e.g. DDAB). In this way one can prepare microemulsions with FA which have essentially the same properties as those in which water acts as the polar solvent. As for aqueous systems, a complete wetting $\rightarrow$ partial non-wetting transition is observed as one proceeds from weakly to strongly structured mixtures. The latter topic has recently been thoroughly studied by small angle neutron scattering [39॰]; the data obtained were in agreement with theoretical considerations [1 ••].

\section{Gaussian curvature}

Up to this point the Gaussian curvature $K=c_{1} c_{2}$ has not been discussed explicitly, although it may be an important quantity (Eq 4). Also, the geometric description [40 $0^{\circ}$ of surfactant structures may be incomplete in some cases, for example in the case of the swelling of sponge-like bicontinuous mesophases of bilayers of surfactant (or lipid) in water $\left[2^{\circ}\right]$, or bicontinuous microemulsions [41 ${ }^{\circ}$. Direct measurement of the Gaussian curvature by scattering is in principle possible, but is rather difficult experimentally $\left[41^{\bullet}\right]$.

\section{Conclusions}

Evidence of a strong correlation of the phase behavior of water-oil-surfactant systems with the variation of the mean curvatures of the internal interface in microemulsions has been re-emphasized. For non-ionic surfactants of the $C_{-j} E_{j}$ type, one finds that the mean interfacial curvature $H$ changes monotonically with temperature from positive (Winsor I) to negative (Winsor II), passing through zero for bicontinuous microemulsions (IVinsor III), where these contain equal volume fractions of water and oil. Furthermore, it is of importance to realize that the interfacial tension between bulk water- and oil-rich phases passes through a characteristic minimum, because at the same time the length scale in the microemulsion reaches a maximum. The maximum occurs because the spontaneous curvature passes zero. Very recently, the latent heat of spontaneous curvature-induced lamellar $\rightarrow$ microemulsion transitions has been measured 
and quantitatively explained $\left[42^{\circ}\right]$ in support of the notion of a bending energy driving and controlling phase behavior and interfacial properties. The effect of temperature for the non-ionics may be replaced by adding fourth and fifth components, such as cosurfactants or salt, or by varying other parameters [43\%]. By mixing surfactants one may tailor the surfactant curvature at a prescribed temperature. These trends are seen to be relevant for emulsions and also for mesophases.

\section{References and recommended reading}

Papers of particular interest, published within the annual period of review, have been highlighted as:

- of special interest

$\bullet$ of outstanding interest

\section{Gompper G, Schick M: Self-assembling amphiphilic systems.} -. Phase Transitions Crit Phenom 1994, 16:1-176.

A review of the properties of water-oil-amphiphile mixtures, which includes more than 380 references. Phase behavior, interfacial properties, and structure are examined in connection with theoretical concepts (e.g. Ginzburg-Landau theory). Fluid membrane bending elasticity, lamellar phases, vesicles, amphiphile bilayers, capillary waves, and wetting transitions are also discussed.

\section{Strey R: Microstructure and interfacial curvature. Colloid Polym}

-. Sci 1994, 272:1005-1019

A review (including 85 references) of the typical phase behavior of microemulsion systems undergoing phase inversion. The occurring microstructures are visualized by freeze-fracture electron microscopy and the corresponding domain sizes are quantified by small angle neutron scattering. From the variations of the domain sizes the mean and Gaussian curvatures of the interfacial film with temperature are determined. The origin of the interfacial tension minimum between bulk water-and oil-rich phases is explained by the temperature dependent variation of the spontaneous curvature.

3. Winsor PA: Solvent Properties of Amphiphilic Compounds. Butterworth: London; 1954.

4. Kahlweit $M$, Strey R: Phase behavior of ternary systems of the type $\mathrm{H}_{2} \mathrm{O}$-oil-non-ionic amphiphile (microemulsions). Angew Chem Int Ed 1985, 24:654-668.

5. Kahlweit $M$, Strey R, Firman P: Search for tricritical points in ternary systems: water-oil-non-ionic amphiphile. J Phys Chem 1986, 90:671-677.

6. Kahlweit M, Strey R, Haase D, Firman P: Properties of the threephase bodies in $\mathrm{H}_{2} \mathrm{O}$-oil-non-ionic amphiphile mixtures. Langmuir 1988, 4:785-790.

7. Kahlweit $M$, Strey R: Phase behavior of quinary mixtures of the type $\mathrm{H}_{2} \mathrm{O}$-oil-non-ionic amphiphile-ionic amphiphile-salt $J$ Phys Chem 1988, 92:1557-1563.

8. Kunieda $H_{1}$ Nakamura $N$ : Phase behavior and self-organizing - $\quad$ structures in sucrose alkanoate solutions. Fragrance $J 1995$, 23:32-38.

A review of the three-phase behavior of sucrose alkanoates or their mixfures with ordinary polyoxyethylene-type non-ionic surfactants in water and oil. Temperature insensitive microemulsions can be formed in sucrose monoalkanoate-cosurfactant systems. The formation and structure of lamellar liquid crystals and reverse vesicles are also discussed.

9. Anton R, Mosquera F, Oduber M: Anionic-non-ionic surfactant mixture to attain emulsion insensitivity to temperature. Prog Colloid Polym Sci 1995, 98:85-88.

10. Kahlweit $M$, Strey $R$, Firman $P$, Haase $D$, Jen J, Schomaecker $R$ : General patterns of the phase behavior of mixtures of $\mathrm{H}_{2} \mathrm{O}$, non-polar solvents, amphiphiles, and electrolytes. 1. Langmuir 1988, 4:499-511.

11. Kahlweit $M$, Strey $R$, Schomäcker $R$, Haase $D$ : General patterns of the phase behavior of mixtures of $\mathrm{H}_{2} \mathrm{O}$, non-polar solvents, amphiphiles, and electrolytes. 2. Langmuir 1989, 5:305-315.

12. Kahlweit $M$, Strey $R$, Busse $G$ : Microemulsions - a qualitative thermodynamic approach. J Phys Chem 1990, 94:3881-3894.

13. Helfrich $W$ : Elastic properties of lipid bilayers: theory and possible experiments. Z Naturforsch C 1973, 8:693-705.
14. Leitao H, Somoza AM, Telo Da Gama MM, Sottmann T, Strey - R: Scaling of the interfacial tension: a phenomenological description. J Chem Phys 1996, in press.

A striking scaling behaviour of the ultra-low interfacial tension of microemulsions considers a quadratic elastic free energy and internal stresses in the membrane which break the symmetry between the two principal curvatures. This analysis provides a straightforward method of obtaining estimates of the bending elastic constants from surface tension measurements. Experiments confirming the theoretical picture are presented, and values for a variety of systems are obtained. As a result of this analysis, the sign in front of the second term in Equation 4 has changed from negative [ $\left.2^{\circ} \cdot\right]$ to positive to permit negative values of $\bar{\kappa}$.

15. Kunieda $H$, Nakano A, Pes MA: Effect of oil on the solubilization

-. in microemulsion systems Including non-ionic surfactant mixtures. Langmuir 1995 11:3302-3306.

A very useful discussion of the effect of partitioning of non-ionic surfactants between oil domains and the interface inside the microemulsion phase in terms of mass balance. The mixing ratio of the surfactants at the interface is obtained from the geometric relation of the three-phase tie triangle in the composition tetrahedron.

16. Penders MHGM, Strey R: Phase behavior of the quaternary -. system $\mathrm{H}_{2} \mathrm{O} / \mathrm{n}$-octane/ $\mathrm{C}_{8} \mathrm{E}_{5} / \mathrm{n}$-octanol: role of the alcohol in microemulsions. J Phys Chem 1995, 99:10313-10318.

The effect of adding n-octanol $\left(\mathrm{C}_{8} \mathrm{E}_{0}\right)$ to the ternary microemulsion system water-n-octane-pentaethylene glycol mono-n-octyl ether $\left(C_{8} E_{5}\right)$ is shown to be a tuning of the spontaneous curvature of mixed amphiphilic film. Moreover, the amphiphilic mixture $\left(\mathrm{C}_{8} \mathrm{E}_{5}+\mathrm{C}_{8} \mathrm{E}_{0}\right)$ displays synergism, as seen from measurements of the interfacial tension between bulk water- and oil-rich phases. The interfacial tension minimum is obtainable by changing either the temperature or the $\mathrm{C}_{8} \mathrm{E}_{0}$ content.

17 Jonströmer $M$, Strey R: Nonionic bilayers in dilute solutions: effect of additives. I Phys Chem 1992, 96:5993-6000.

18. Penders MHGM Strey R: Lamellar and $L_{3}$ phases in the 'simple' - $\mathrm{H}_{2} \mathrm{O}-\mathrm{C}_{8} \mathrm{E}_{5}-1$-octanol system: evidence of synergism. J Phys Chem 1995, 99:6091-6095

The paper illustrates how composition-temperature sections can be used to study complex phase behavior. A surprising synergism and the occurrence of dilute $L_{3}$ and lamellar phase are both reported.

19. Adamy ST: Phase studies of water/alcohol/1-octyl-2pyrrolidone/alkane systems. Langmuir 1995, 11:3269-3271.

20. Leaver MS, Olsson U, Wennerstroem $H$, Strey R, Wuerz U: Phase - behavior and structure in a non-ionic surfactent-oil-water mixture. $J$ Chem Soc Faraday Trans 1995, 91:4269-4274.

The mean curvature of the polar/apolar interface in structures of normal spheres, planar bilayers and cylinders is observed as a function of decreasing water concentration at fixed temperature. At fixed surfactant and oil concentration, the mean curvature progressively alters from positive values in the low temperature microemulsion phase to negative mean curvature at high temperature in the $L_{3}$ phase, with mean curvature passing through zero in the intermediate lamellar phase. A nice example of the connection of phase behavior and mean curvature.

21. DeGeyer A: Phase behavior of surfactant-alcohol-oil-water cubic liquid ciystals. Prog Colloid Polym Sci 1993, 93:76-80.

22. Engblom J, Hyde ST: On the swelling of bicontinuous lyotropic - mesophases. J Phys // 1995 5:171-190.

The swelling of sponge-like bicontinuous mesophases of bilayers of surfactant is compared with swelling behavior of disconnected sheet-like, rodlike and globular aggregates. Accurate scaling laws are compared with experimental data of bicontinuous cubic phases in the binary glycerol monooleate-water system and the pseudo-binary DDAB-cyclohexane-water system. An interesting study containing useful formulae.

23. Foerster $\mathrm{T}$, Von Rybinski $W$, Wadle $A$ : Influence of

- microemulsion phases on the preparation of fine-disperse emulsions. Adv Colloid Interface Sci 1995, 58:119-149.

Emulsions in cosmetics and technical applications are obtained by temperature-induced phase inversion from an $(O N W)(O N W)$ to a $(W / O)$ (W/O) type. The ultralow interfacial tension enables finely dispersed emul. sions with long-term stability to be produced by phase inversion without high mechanical energy input. This review presents important basic properties and certain rules allowing the preselection of optimal emulsification routes and optimal emulsifier/oil combinations for cosmetic emulsions.

24. Kabalnov $A$, Wennerström $H$ : Macroemulsion stability: the *. oriented wedge theory revisited. Langmuir 1996 12:276-292. Macroemulsion type and stability depend on the bending elasticity of the surfactant monolayer at the oil/water interface. Spontaneous curvatures explain the effect of the monolayer bending properties on the macroemulsion stability. A model reproducing the macroemulsion stability sequence $\mathrm{ON}$ emulsion $\rightarrow$ emulsion breakage $\rightarrow W / O$ emulsion is compared with experimental observations in polyethoxylated non-ionic surfactant-oil-water mixtures. Possible extensions of the model to other systems are discussed. 
25. Davis HT: Factors determining emulsion type:

.. hydrophile-lipophile balance and beyond. Colloid Surf A 1994, 91:9-24.

The author discusses the advantage of basing the hydrophile-lipophile balance (HLB) on the phase-inversion temperature (PIT). More generally, the effect of cosurfactants, frequently needed for stable emulsions, is accounted for as a shift in the PIT, as one example of field variables at which the optimal microemulsion occurs providing an optimal point (OP) that can serve as the HLB for a given system. The relationship between the generic phase behavior of surfactant, oil and water systems and the curvature properties of surfactant interfacial films is also discussed.

26. Lusvardi KM, Schubert KV, Kaler EW: Phase behavior and - microstructure of polymerizable microemulsions. Langmuir 1995, 11:4728-4734.

Microstructure analysis using small angle neutron scattering and phase behavior of polymerizable alkyl methacrylates in aqueous solution in mixture with the cationic surfactants DTAB and DDAB show that $O W$ microemulsions form and that the shape of the aggregates depends on the DTAB/DDAB ratio and the oil content.

\section{Schwuger MJ, Stickdorn K, Schomaecker R: Microemulsions in} - technical processes. Chem Rev 1995, 95:849-864.

A review of the basic properties of microemulsions and their relationships to some established applications such as enhanced oil recovery, liquid-liquid extraction, washing, and textile finishing. Nanoparticle preparation, biochemical, electrochemical, catalytic and organic reactions and a comparison with phase transfer catalysis are also discussed.

28. Eastoe J, Cox AR: Formation of PbS nanoclusters using reversed micelles of lead and sodium Aerosol-OT. Colloid Surf A 1995, 101:63-76.

29. Oldfield C: Enzymes in water-in-oil microemulsions ('reversed - micelles'): principles and applications. Biotechnol Genet Eng Rev 1994, 12:255-327.

A review of enzyme reactions in W/O microemulsions (reverse micelles). Topics discussed include phase behavior, structure, and dynamics of microemulsions, the properties and behavior of microemulsified enzymes, recent ad vances in process engineering of microemulsions with a view to industrial scale biotransformations, and new research, trends and future prospects.

30. Karayigitoglu CF, Kommareddi N, Gonzalez RD, John VT, McPherson GL, Akkara JA, Kaplan DL: The morphology of phenolic polymers enzymatically synthesized in surfactant microstructures. Mater Sci Eng C 1995, 2:165-171.

31. Backlund $S$, Eriksson $F$, Kanerva LT, Rantala M: Selective enzymic reactions using microemulsion-based gels. Colloid Surf $B$ 1995, 4:121-127.

32. Singh CP, Shah DO: Lipase-catalyzed esterification in monolayers and microemulsions. Colloid Surf $A 1993$ 77:219-224.

33. Salager $J$, Marquez N, Anton RE, Graciaa A, Lachaise

- J: Retrograde transition in the phase behavior of surfactant-oil-water systems produced by an alcohol scan. Langmuir 1995, 11:37-41.

The anomalous phase behavior of a commericial non-ionic polyethoxylated surfactant, $n$-heptane, $\mathrm{H}_{2} \mathrm{O}$, and $n$-pentanol is analyzed by high pressure liquid chromatography to show the surfactant oligomer partitioning between phases affected by the alcohol content. A retrograde transition is shown to be caused by the strong increase in the partitioning of lipophilic and balanced oligomers into the oil phase, with the interfacial surfactant mixture becoming more hydrophilic. An important mechanism to watch out for in technical grade surfactant mixtures.

34. Fukuda K, Soederman O, Shinoda K, Lindman B: Microemulsions formed by alkyl polyglucosides and an alkyl glycerol ether. Langmuir 1993, 9:2921-2925.
35. Minana-Perez M, Graciaa A, Lachaise J, Salager JL: Solubilization of polar oils with extended surfactants. Colloid Surf $A 1995$ 100:217-224.

36. Eastoe J, Steytler DC, Robinson BH, Heenan RK: Pressureinduced structural changes in water-in-propane microemulsions. J Chem Soc Faraday Trans 1994 90:3121-3127.

37. McFann GJ, Johnston KP: Phase behavior of non-ionic surfactant-oil-water systems containing light alkanes. Langmuir 1993 9:2942-2948.

38. Schubert KV, Busse G, Strey R, Kahlweit M: Microemulsions with formamide as polar solvent. $J$ Phys Chem 1993, 97:248-254.

39. Schubert KV, Strey R, Kline SR, Kaler EW: Small angle neutron - $\quad$ scattering near the Lifshitz lines: transition from weakly structured mixtures to microemulsions. J Chem Phys 1994 101:5343-5355.

The phase behavior, wetting transitions and small angle neutron scattering of water, $n$-alkane, and $n$-alkyl polyglycol ether are determined. An amphiphilicity factor permits visualization of a sequence of roughly parallel surfaces within the three-dimensional composition-temperature space. The extent to which these surfaces move in the one-phase region towards smaller surfactant concentration and intersect there with the body of heterogeneous phases depends on a number of factors discussed.

40. Ciach A, Poniewierski A: Description of the geometrical and - topological structure in amphiphilic systems. Phys. Rev E 1995, 52:596-601.

A general definition of average curvatures of the mean and Gaussian curvatures characterizes globally the geometrical and topological structure of the internal interface. This definition can be applied to both sharp and diffuse oil/water interfaces, in ordered phases and in disordered microemulsions.

41. Lee DD, Chen SH: Geometry of bicontinuous microemulsions - as revealed by SANS and simulations. Nuovo Cimento D-Cond Matt At 1994, 16:1357-1366.

Water-n-octane $-\mathrm{C}_{10} \mathrm{E}_{4}$ microemulsions in both the isotropic and lamellar phases are measured by small angle neutron scattering using hydrogen-deuterium substitution, yielding the mean curvature of the surfactant film as a function of temperature and correlated with the overall phase behavior. A simulation using a Gaussian random field yields the three-dimensional structure of the bicontinuous microemulsion having a surfactant monolayer with zero mean curvature and negative Gaussian curvature.

42. Vollmer $D$, Strey $R$ : Latent heat of spontaneous-curvature- induced lamellar-to-microemulsion transitions. Europhys Lett $1995,32: 693-698$.

Differential scanning microcalorimetric measurements on the temperatureinduced structure transitions from lamellar $\rightarrow$ microemulsion phases show the transition yields large latent heats, which can be understood, if and only if, the (linear) temperature dependence of the spontaneous curvature is taken into account.

\section{Kahlweit M: How to prepare microemulsions at prescribed} - temperature, oil, and brine. J Phys Chem 1995 99:1 281-1284. The author summarizes trends and knowledge from a decade of experiments in his laboratory. Also, for those who want to prepare microemulsions without resorting to the notion of spontaneous curvature and bending energy, a number of rules are given.

44. Sottmann T, Strey R: Shape and similarities of ultra-low Interfacial tension curves in ternary microemulsions of the water-alkane- $C_{i} E_{j}$ type. Ber Bunsenges Phys Chem 1996, 100:237-241. 